\title{
Integrar las TIC al currículo en la educación media
}

Dora Lidia Orjuela Forero ${ }^{1}$

\begin{abstract}
Resumen
Este artículo es el resultado de una investigación que propone una metodología para la integración curricular de las tecnologías de la información y comunicación en el nivel de educación media, mediante un estudio de caso simple que toma como unidad de análisis el grado décimo de una institución educativa y como subunidades de análisis a los directivos, docentes, estudiantes y padres de familia con quienes se desarrolló una estrategia denominada "Las TIC en el aula". La metodología plantea diagnosticar necesidades de integración, establecer programas de capacitación, planear el proceso teniendo en cuenta los intereses de docentes y estudiantes, hacer la implementación de las tecnologías de la información y la comunicación en sus fases elemental, básica, mejorada y experta, de acuerdo con las características institucionales diagnosticadas $\mathrm{y}$, finalmente, evaluar para identificar fortalezas y oportunidades de mejoramiento. Esta metodología ayuda a transformar prácticas de enseñanza, mejorar aprendizajes e impactar positivamente a la comunidad educativa, pasando de relaciones pedagógicas verticales a horizontales y de un currículo tradicional a un currículo integrado mediado por las nuevas tecnologías.
\end{abstract}

Palabras clave: TIC, currículo, integración, metodología, transformación. 


\begin{abstract}
TThis article is the result of a study that proposes a methodology for curriculum integration of information technology and communication in the middle school level, through a simple case study that takes as its unit of analysis at a tenth grade school and as subunits of analysis to managers, teachers, students and parents with whom it was developed a strategy called "ICT in the classroom." The methodology raises diagnose integration needs, establishes training programs, plans the process, taking into account the interests of teachers and students, to the implementation of information technology and communication in stages: elementary, basic, enhanced and expert according to the institutional features eventually diagnosed and evaluated to identify strengths and improvement opportunities. This methodology helps to transform teaching practices, enhance learning and impact positively the educational community, from vertical to horizontal relationships and from a traditional curriculum to an integrated curriculum mediated by new technologies.
\end{abstract}

Key words: ICT, curriculum, integration, methodology, processing.

Recibido: 10 de septiembre de 2010

Aceptado: 28 de octubre de 2010

\title{
Introducción
}

La integración curricular de las TIC es una necesidad en los todos los niveles educativos (preescolar, básica y media), ya que éstas permiten el manejo de la información entre personas y contextos, facilitan la producción de conocimiento y se convierten en herramientas poderosas para el aprendizaje. Es por esto que en la Institución objeto de estudio se desarrolla un estudio de caso simple que toma como unidad de análisis principal el grado décimo de la Institución Educativa Panamericana Puente de Boyacá y como subunidades de análisis a los directivos, docentes, estudiantes y padres de familia

En esta institución se encuentran tecnologías de la información que no son utilizadas por los docentes en su desempeño pedagógico por falta de capacitación en su uso y desconocimiento de estrategias y modelos de integración curricular que logren potenciarlas como medios para apropiar conocimientos y transformar las prácticas de enseñanza. 
Coincidiendo con esta problemática, Pedraza \& Lara (2007) afirman que en la actualidad "las TIC están siendo usadas con mayor frecuencia en la dinámica educativa; por ello es preciso analizar el currículo, reflexionando sobre cómo está cambiando con el uso de las tecnologías, y cómo se están transformando los escenarios educativos, los procesos de formación y de igual manera el quehacer docente". De tal forma que "una vez que se posea la tecnología y los profesores aprendan a usarla, el tema que surge es cómo integrarla al currículo”. Sánchez (2002).

También Hurtado (2002), citando a Gallego \& Alonso (1999), plantea la importancia de integrar las TIC en la dinámica educativa, "ya que su uso va a posibilitar el ajuste a las necesidades educativas de cada usuario, haciendo posible una respuesta educativa de calidad" donde se tenga en cuenta "la avalancha de información o de contenidos de conocimiento disponibles en Internet, el potencial de las TIC para actualizar, transformar y enriquecer, a bajo costo, los ambientes de aprendizaje en los que se educan niños y jóvenes latinoamericanos y la necesidad de desarrollar la competencia en TIC" (FGPU, 2002).

Por esto, se hace necesario proponer una metodología para la integración curricular de las TIC en instituciones de educación media que dé cuenta de los fundamentos conceptuales, el desarrollo y evaluación de estrategias de integración curricular, los elementos de la metodología de integración y el análisis de su incidencia en los actores del currículo.

Las sugerencias metodológicas en el marco de un currículo flexible y articulado de acuerdo con los principios y elementos establecidos en el Proyecto Educativo Institucional (PEI), permiten evidenciar procesos de integración curricular de las TIC en la educación media.

\section{Referentes teóricos}

La integración curricular de las TIC es definida por Sánchez (2002), basado en los aportes de Grabe \& Grabe (1996), Merrill et al. (1996) y Gros (2000) como "el proceso de hacerlas enteramente parte del currículum, como parte de un todo, permeándolas con los principios educativos y la didáctica que conforman el engranaje del aprender"; es "el ensamble de las mismas dentro del currículo", cuyo "propósito es la actividad de aprendizaje, la acción pedagógica". Así, las TIC son herramientas que se utilizan para fines curriculares, para apoyar una disciplina o un contenido y para estimular el desarrollo de aprendizajes 
de orden superior, tornándose invisibles para que el docente y el estudiante se apropien de ellas y las utilicen en un marco situado del aprender.

Según Sánchez (2002), Merrill et al. (1996), Escudero (1995), Dede (2000) y Gros (2000), integrar curricularmente las TIC implica utilizarlas transparentemente en el aula: para apoyar las clases, para aprender el contenido de una disciplina o una asignatura integrada, para planificar estrategias que faciliten la construcción del aprender, en la articulación pedagógica donde pase desapercibida la tecnología para dar visibilidad al aprender.

Existen seis modelos de integración curricular de las TIC propuestos por Sánchez (2002): anidado, tejido, enroscado, integrado, inmerso y en red, partiendo de los principios de currículo integrado explicado y sustentado por Jacobs (1991) y Fogarty (1991).

El modelo anidado propone que "en una asignatura el profesor estimule el trabajo de distintas habilidades, de pensamiento, social y de contenido específico"; el modelo tejido implica tomar "un tema relevante y tejerlo con otros contenidos y disciplinas, los aprendices utilizan el tema para examinar conceptos e ideas con el apoyo de las TIC"; el modelo enroscado sugiere, como su nombre lo indica, "enroscar habilidades sociales, de pensamiento, inteligencias múltiples, tecnología y de estudio a través de varias disciplinas"; el modelo integrado se da al "unir asignaturas en la búsqueda de superposiciones de conceptos e ideas, utilizando las TIC"; el modelo inmerso propone que las "asignaturas sean parte del pénsum del aprendiz, filtrando el contenido con el apoyo de las TIC, llegando a estar inmerso en su propia experiencia" y finalmente el modelo en red en donde "el aprendiz realiza un filtrado de su aprendizaje y genera conexiones internas que lo llevan a interacciones con redes externas de expertos en áreas relacionadas, utilizando las TIC".

También Sánchez (2002) plantea tres niveles de integración de las TIC: aprendizaje, uso e integración. El primero implica vencer el miedo y descubrir las potencialidades de las TIC sin ningún uso educativo; el segundo, conocer y usar las TIC para diversas tareas, pero sin un propósito curricular claro; y el tercero, integrarlas al currículum para un fin educativo explícito.

La Fundación Gabriel Piedrahíta Uribe - FGPU (2008) plantea que el proceso de integración curricular de las TIC debe tener en cuenta: dirección institucional, infraestructura TIC, coordinación y docencia TIC, docentes de otras áreas y recursos digitales.

De igual forma sugiere que durante el desarrollo profesional de los docentes se den los niveles de: 1) pre-integración, cuando se "hace uso de las TIC para incrementar su productividad personal"; 2) integración dirigida, cuando se utilizan las TIC "como herramienta de instrucción programada para que los estudiantes se 
entrenen con tutoriales y software de ejercicio y práctica"; 3 ) integración básica, cuando se "emplean las TIC para elaborar mejores materiales para sus estudiantes y volver más interesantes las clases de las asignaturas a su cargo"; 4) integración media, cuando se solicita a los estudiantes utilizar las TIC para realizar trabajos de clase"; 5) integración avanzada, cuando se "aprovechan las TIC y metodologías de aprendizaje activo para realizar proyectos de clase enfocados en el currículo con el objeto de lograr mejoras en el aprendizaje de los estudiantes" y 6) integración experta, cuando "diseñan y utilizan ambientes constructivistas de aprendizaje enriquecidos por TIC".

Gutiérrez (2007) propone integrar las TIC al currículo creando asignaturas específicas para el estudio de los medios y considerarlas como contenido transversal al currículo. Herrera (2008) sugiere un modelo que parte de la filosofía de la integración y llega a la reflexión y evaluación.

Hurtado (2002) sugiere cuatro premisas necesarias para abordar con éxito la integración curricular de las tecnologías de la información y la comunicación: 1) la formación del profesorado en las $\mathrm{TIC} ; 2$ ) el conocimiento de los programas educativos; 3) la inclusión de éstos recursos en la planeación escolar y 4) la organización de los centros educativos para optimizar estos recursos.

Pedraza \& Lara (2007) como conclusión de un grupo de estudios de la red virtual de tutores, sugieren que se deben tener en cuenta elementos para diseñar los lineamientos para la integración de las TIC al currículo, que partan de la familiarización con el proyecto, el análisis y la evaluación de lo existente, la selección de tecnologías, la integración del diseño, la instrucción, la construcción, y lleguen a la implementación, el monitoreo y la adaptación.

Para tener mayor claridad se hace necesario conceptualizar TIC, currículo e integración curricular.

Las TIC, según el programa de las Naciones Unidas para el Desarrollo (2002), son "un conjunto de tecnologías e instrumentos para procesar varios tipos información". Para el Plan Nacional de Tecnologías de la Información y la Comunicación, en Colombia son "un conjunto de herramientas y equipos". Camargo (2007) afirma "que están constituidas por las tecnologías modernas de la información". Márquez (2000) sostiene que son "la informática y sus tecnologías asociadas, medios de comunicación de todo tipo" y plantea aportaciones a la educación en cuanto a "facilidad de acceso a la información, instrumentos, canales de comunicación, almacenamiento de información, interactividad, homogenización de códigos e instrumento cognitivo". Herrera (2008) afirma que son "herramientas para estimular el desarrollo de aprendizajes de orden superior".

Las TIC son un conjunto de tecnologías, instrumentos, procesos, medios, herramientas tecnológicas, canales de comunicación y creaciones de la sociedad 
en torno al manejo de la información entre personas y contextos que facilitan la producción de conocimiento en diferentes ámbitos.

Las TIC son un cuerpo de conocimiento que se está construyendo permanentemente y están presentes en todos los sectores de las sociedad. Organizaciones mundiales como la Unesco, las Naciones Unidas, la Sociedad Internacional de Tecnología en Educación (ISTE), la Organización de los Estados Iberoamericanos para la Educación, la Ciencia y la Cultura (OEI) y los programas de educación de los diferentes países, promueven y plantean políticas y estándares en busca de la igualdad de condiciones en cuanto al acceso y desarrollo de competencias básicas para desenvolverse en la nueva sociedad de la información y el conocimiento.

En Colombia se plantean políticas educativas en torno de las TIC que se materializan y fundamentan desde el Plan Nacional de Tecnologías de la Información y la Comunicación (PNTIC) y el Plan Nacional de Educación (PNDE), los cuales sugieren su incorporación en el currículo institucional.

Sobre el currículo han conceptualizado autores como Johnson (1967), Gimeno (1981), Porlan (1993), Stenhouse (1991), Sánchez (2002) y (Montenegro 2005), entre otros, y la Ley General de Educación en Colombia con su decreto reglamentario 1860 de 1994. Todos ellos son referentes que permiten definir el currículo como un todo articulado y flexible que ayuda a la institución educativa a recorrer el camino trazado en el PEI, que refleja los principios e intereses de sus actores y se materializa en los planes de estudios, metodologías $\mathrm{y}$ formas de integración con recursos y otros programas.

El currículo puede ser llevado a la práctica desde diversas formas; de ahí que se hable de currículo oculto, tradicional, abierto, flexible e integrado. Para efectos de este trabajo, se tomaron diversos aportes desde las miradas de Ortiz (2006), Jacobs (1991) y Fogarty (1991), definiéndose como un todo articulado que trabaja con la unidad de disciplinas, que rompe los límites de las áreas, que permite que se organicen los aprendizajes comunes en espacios donde se comparten destrezas y conceptos para dar sentido a los contenidos curriculares a través de temas generativos y metas unificadas. Se contribuye así a que el aprendizaje de los estudiantes sea significativo, dado que hace conexiones que le permiten apropiarse de los nuevos conocimientos, propiciando una enseñanza activa y flexible y convirtiendo al docente en un facilitador de ese aprendizaje.

En otras palabras, la integración curricular se logra al unir, combinar, complementar, articular y organizar una de serie de elementos para construir un todo que tenga una función conjunta, con el fin de facilitar el aprendizaje significativo de los estudiantes y la transformación en las prácticas de enseñanza de los docentes, hechos que redundan también en la transformación de la sociedad. 


\section{Metodología}

El tipo de investigación está enmarcado en el paradigma cualitativo, el enfoque histórico-hermenéutico y el diseño de estudio de caso simple tomando como unidad de análisis principal a los directivos, docentes, estudiantes y padres de familia del grado décimo de la Institución Educativa Panamericana Puente de Boyacá.

Esta institución presenta dificultades en los procesos de aprendizaje por parte de los estudiantes. Hace falta capacitación de los docentes en el uso de las TIC; los recursos tecnológicos están subutilizados, hay resistencias al cambio de las dinámicas institucionales, como también interés en la incorporación de las TIC a los procesos pedagógicos por parte de los actores del currículo.

Ante esta problemática se plantean interrogantes como: ¿cuál es la fundamentación conceptual que establece la integración curricular de las TIC?; ¿qué es la integración curricular de las TIC y qué implica este proceso?; ¿cómo integrar las TIC a una institución que ofrezca los niveles de básica y media?, y ¿cuál es el impacto del proceso de integración de las TIC en esta institución educativa?

Para resolver los interrogantes se indaga en estas fuentes: PEI, plan de estudios institucional, planes de área, estándares de competencias, preparadores de clase, proyectos existentes, experiencias de docentes, leyes y documentos sobre políticas de TIC. Además se utilizan como instrumentos la entrevista a docentes y estudiantes, la matriz de revisión de planes de área, la matriz de contenidos y estrategias, el diario de campo y la observación directa.

Se desarrolla en tres fases: 1) la prueba piloto ejecutada con la estrategia denominada "filomáticos", que tenía como propósito motivar a los docentes y estudiantes de la institución para integrar las TIC al currículo e identificar algunos aspectos a tener en cuenta en el proceso de integración; 2) la aplicación del proceso de integración curricular de las TIC, ejecutada con la estrategia denominada "las TIC en el aula" y con base en los resultados de la prueba piloto, los niveles de integración de las TIC y los modelos de ICT; 3) la evaluación para identificar los resultados, contrastar con el marco teórico, describir el proceso y proponer una metodología de integración curricular de las TIC en los niveles de básica y media.

\section{Propuesta metodológica para integrar las tic al currículo}

La propuesta metodológica para integrar las TIC al currículo requiere que se defina como un proceso, un ensamblaje, una articulación pedagógica con intencionalidad curricular que tiene como propósito aprender contenidos disciplinantes y desarrollar competencias en el manejo de las TIC. De tal forma, las tecnologías de la información y la comunicación son el vehículo para lograr este propósito y no el fin de la integración, incidiendo en el aprendizaje, la enseñanza, la transferencia 
al contexto, la solución de problemas o la inmersión en un nuevo entorno social de información y conocimiento.

En el marco del Proyecto Educativo Institucional (PEI), debe evidenciarse la voluntad para combinar la tecnología, el aprendizaje y la enseñanza en aras de una experiencia productiva que mueva a los docentes y estudiantes a cambiar sus paradigmas y estructuras, para que se dé una asimilación y acomodación del currículo sobre las TIC.

Desde el PEI se debe incentivar la integración de las TIC en el currículo como forma de propiciar el cambio de un currículo tradicional y vertical a uno integrado horizontal "dentro de una disciplina, a través de las disciplinas o dentro de la mente del aprendiz", según Fogarty (1991). Hay que utilizar diferentes elementos ya existentes como las estrategias metodológicas de los docentes, las particularidades de los contenidos, la disponibilidad de los recursos, la capacitación en uso de las TIC y, lo más importante, la motivación de docentes y estudiantes.

Es importante que el proceso de integración sea gradual, de acuerdo con los niveles de interés y motivación, principalmente con los docentes quienes, por temor a romper paradigmas y por desconocer las nuevas tecnologías, obstaculizan muchas veces los aprendizajes de los estudiantes y la aplicación de nuevas formas de trabajo interdisciplinario y de integración de otros medios como las TIC para desarrollar sus procesos de enseñanza. Por esta razón, se debe partir no sólo de los intereses de los docentes, de sus estrategias y de los elementos de control con los que interactúan, sino también del interés de los estudiantes y de los medios tecnológicos a los que acceden permanentemente, con el fin de facilitar su desempeño implementando nuevas estrategias, más dinamizantes e interactivas, que interesen y faciliten el trabajo en clase en vez de que se conviertan en una nueva actividad para aumentar su carga laboral.

La propuesta metodológica sugiere, para integrar curricularmente las TIC en una institución educativa que ofrezca los niveles de educación básica y media, etapas secuenciales así: Diagnosticar, capacitar, planear, desarrollar y evaluar (Ilustración 1).

\section{- Diagnosticar}

Al diagnosticar, se pueden encontrar diversas necesidades de integración curricular de las TIC, de acuerdo con el papel que asume cada uno de los actores del currículo. Los docentes, por ejemplo, pueden mejorar el aprendizaje de los contenidos, mejorar los resultados de las evaluaciones externas Icfes y Saber, desarrollar actividades de administración del currículo, permitir el trabajo interdisciplinario y en equipo, evaluar a sus estudiantes, capacitarse en TIC para desarrollar sus competencias, tener buenas fuentes de consulta o simplemente, facilitar su trabajo. 


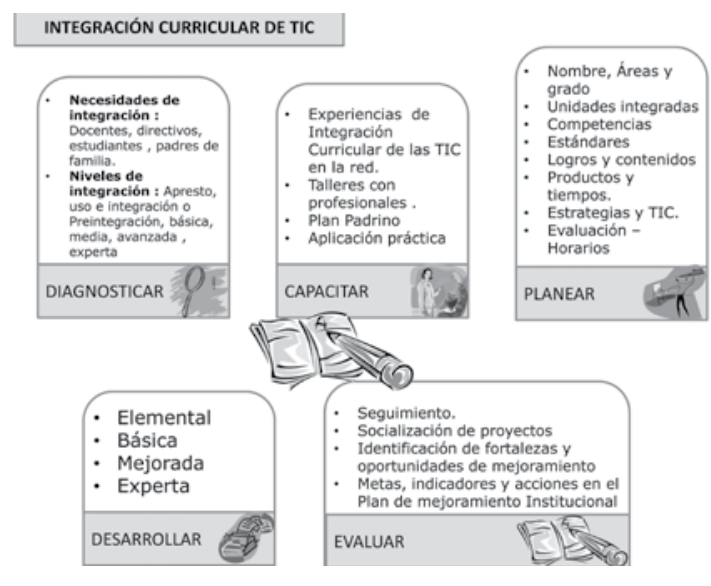

Ilustración 1. Metodología de integración curricular de las TIC

Las necesidades de los estudiantes pueden ser: aprender fácil y rápidamente, divertirse, comunicarse a través de redes sociales, utilizar videojuegos, trabajar con imágenes y sonido, evidenciar la interactividad, tener clases agradables y dinámicas o simplemente, estar a la vanguardia de la tecnología descubriendo nuevas usos de las TIC.

Los directivos pueden contribuir a optimizar los recursos, cumplir con lo planeado en el PEI, proponer alternativas de mejoramiento, fomentar la investigación, capacitar a docentes, mejorar la calidad, etc., de acuerdo con las características de la institución. Las necesidades de los padres pueden ser que sus hijos estén al día en los avances tecnológicos, que aprendan y así tengan mejores oportunidades laborales.

De igual forma se deben identificar los niveles de integración en los que se encuentran los actores del currículo, ya sean estos desde el uso propuesto por Sánchez (2002) o desde el desempeño profesional propuesto por la FGPU (2008).

\section{- Capacitar}

La segunda etapa de la metodología propuesta es la capacitación tanto a estudiantes como a docentes. Para los primeros se hace en el tiempo dedicado al área de informática y con la coordinación del encargado del área. Para los docentes, debe partir de las necesidades de integración diagnosticadas y de la identificación de niveles de integración en cada uno y en el currículo, que priorizadas ofrecen una guía para empezar a capacitarse, aprovechando programas de capacitación en TIC ofrecidos por el Ministerio de Educación Nacional y las Secretarias de Educación o por estrategias definidas por la institución como aplicación de las políticas de cualificación docente determinadas en el PEI. 
Las estrategias de capacitación que se pueden optar son: dar a conocer experiencias de integración de TIC al currículo publicadas en portales corporativos como el MEN, Colombia aprende, Computadores para Educar, Expociencia Educativa; desarrollar actividades prácticas y talleres con profesionales en TIC; plantear el plan padrino en el que el estudiante asesora al docente en el uso de la herramienta y aplicación práctica durante el quehacer pedagógico y el desarrollo de los procesos de cada una de las gestiones en las que está involucrado el docente.

\section{- Planear}

La tercera etapa de la ICT hace referencia a la planeación concertada con todos los actores del currículo, empezando a trabajar gradualmente con los docentes más motivados e interesados hasta lograr la participación de todo el equipo de trabajo. Para este proceso se sugiere una jornada de planeación que debe registrar: nombre de la estrategia, áreas y grados, temas integradores concertados, problemas de investigación o unidades integradas que puedan trabajarse transversalmente, competencias a desarrollar, estándares, logros a alcanzar, contenidos generales que se articulan, productos a entregar, tiempos, estrategias metodológicas y pedagógicas a utilizar, recursos y herramientas TIC, formas de evaluación, cronograma y horario de aplicación de la estrategia de integración curricular de las TIC.

\section{- Desarrollar}

Cuando se encuentran planeadas las unidades integradas, se procede a la cuarta etapa que consiste en desarrollar las estrategias de integración curricular de las TIC utilizando modelos apropiados. Éstas pueden darse en cuatro fases consecutivas: elemental, básica, mejorada y experta según el diagnóstico, el avance en la capacitación y el tiempo de maduración del proceso de integración hasta llegar a su articulación definitiva en el currículo.

La fase elemental se desarrolla cuando el docente es diagnosticado en un nivel de integración anidado. Para este caso se recomienda utilizar el modelo curricular de las TIC seleccionado y su adaptación con éstas en la respectiva área con el apoyo permanente del docente de informática o del coordinador si existe. Esto se hace con el fin de empezar a reconocer la potencialidad de las TIC en el proceso pedagógico y liberar temores en el momento de integrarlas.

La fase básica se aplica cuando se observa en la institución que el docente en su desarrollo profesional hace uso de las TIC para incrementar su productividad personal y para elaborar mejores materiales para sus estudiantes (FGPU, 2008) pero sin un propósito curricular claro. En este caso se sugiere utilizar el modelo de integración tejido uniendo dos o tres áreas disciplinares y las TIC con unidades integradas básicas que motiven a dar los primeros pasos de integración curricular de las TIC a través de las disciplinas. 
La fase mejorada ocurre cuando se diagnostica en la institución el nivel de integración; es decir, cuando el docente solicita a los estudiantes que utilicen las TIC para realizar trabajos de clase, que las aprovechen para mejorar sus aprendizaje y las integren con un fin educativo. En este caso se sugiere utilizar el modelo tejido, enroscado e integrado para que el estudiante construya aprendizajes desde los contenidos de varias disciplinas con un tema integrador y el apoyo de las TIC, desarrollando habilidades de pensamiento y competencias desde la mirada de varias áreas.

La última fase del desarrollo del proceso de integración, y a la que se espera llegar, es la experta. A esta instancia se llega cuando el proceso de integración curricular de las TIC está implementado en la institución educativa y lleva un tiempo de maduración, reflejándose en el uso y diseño que el docente hace de ambientes constructivistas de aprendizaje enriquecidos por las TIC, según Jonassen (1996), por ser activos, constructivos, colaborativos, intencionales, complejos, contextuales, conversacionales y reflexivos. Por ser la última fase, se llega a desarrollar las competencias en TIC para estudiantes, docentes y directivos, estimulando la creatividad y el pensamiento crítico.

Aquí se sugiere utilizar los modelos inmersos y en red con el fin de proponer estrategias para que el estudiante construya su aprendizaje con base en conexiones internas y filtrados de contenidos, interactuando con redes externas. Tanto el docente como el estudiante logran ser expertos en el uso de las TIC para utilizarlas como herramientas estratégicas de enseñanza y de aprendizaje en un corto tiempo.

\section{- Evaluar}

A través de la evaluación se mejora la calidad de la educación en la institución educativa porque se identifican fortalezas y oportunidades de mejoramiento que permiten plantear acciones para que la integración curricular realmente permee el currículo.

\section{Resultados}

Para analizar el impacto de la integración curricular de las TIC en las unidades de análisis establecidas (estudiantes, docentes, directivos y padres de familia), se empieza por hacer una analogía en términos de "nativos", "inmigrantes" y "exiliados" digitales propuesta por Cabello (2006). En cada uno de ellos se generan "transformaciones de acuerdo a las representaciones del mundo de la escuela y la vida cotidiana, porque no todos participan homogéneamente en entornos tecnoculturales donde se encuentran dispositivos técnicos diferentes" y los elementos que hacen parte del currículo. 
Las transformaciones generadas por la integración de las TIC en las prácticas pedagógicas del docente lo concientizan de la transversalidad y la puesta en escena de la horizontalidad en la relación pedagógica en pro de la construcción significativa de conocimientos. El docente, por tanto, se convierte en "inmigrante digital" que rompe barreras al identificar las fortalezas y proponer alternativas de mejoramiento.

En cuanto a los estudiantes, "nativos digitales" que viven en un medio donde es cotidiano el lenguaje de las TIC, al entrar en contacto con el mundo a través de la tecnología potencian con mayor creatividad los contenidos que "antes eran aburridos" y logran organizar información con video, imagen, texto y mapas conceptuales, mejorando de esta forma la comprensión de contenidos y desarrollando competencias. No obstante, el computador e Internet pueden ser distractores en muchos casos, por lo que se requiere analizar y proponer estrategias que ayuden a aprender y a aprovechar la versatilidad de los estudiantes al utilizar los diversos medios tecnológicos.

Los directivos son entonces los encargados de potenciar las "migraciones digitales" con procesos donde se ponga a disposición un conjunto de condiciones, orientaciones, acciones y recursos. Este conjunto, tratado de manera planificada, explícita, sistemática y recurrente, contribuye a que las personas puedan relacionarse del modo más fluido posible, aprovechando las tecnologías digitales, apropiándose de ellas en sus múltiples posibilidades, integrándolas en sus planes de acción e interacción, evaluando sus alcances y limitaciones y tomando nota de sus cambios" (Cabello y Aprea, 2004).

Se puede decir que nativos, inmigrantes, exiliados o potenciadores de migraciones digitales, estando unidos, fortalecen las construcciones de conocimientos en torno a las unidades integradas, ayudan a fomentar la horizontalidad de la relación pedagógica, la puesta en escena del diálogo de saberes, la transversalidad y la disminución de la brecha digital que los separa.

A pesar de todo, se han fortalecido las prácticas institucionales; se han hallado caminos para cambiar metodologías, permitir el trabajo en equipo y propiciar espacios para el aprendizaje dentro y fuera del aula. Además, se ha generado un ambiente escolar agradable, de confianza, de comunicación entre docentes y de colaboración, en donde el estudiante se siente motivado por aprender áreas y contenidos que a veces parecían aburridos.

Siendo los directivos, estudiantes, docentes y padres de familia las unidades de análisis, se entrevistan y se categoriza la información para mostrar cómo la integración curricular de las TIC transformó el aprendizaje, las prácticas de enseñanza y causó impacto en cada uno de los actores del currículo. 
Los docentes muestran en sus opiniones que la integración curricular de las TIC benefició a los estudiantes porque mejoraron sus aprendizajes en un $67 \%$, se motivaron para el trabajo con otras áreas del conocimiento en un $22 \%$ y facilitaron el trabajo en equipo en un $11 \%$ (gráfico 1).

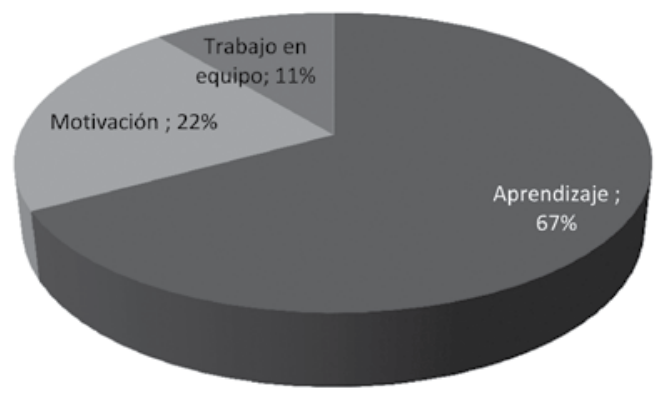

Figura 1. Beneficios para el estudiante. Datos obtenidos de entrevista a docentes

Según los mismos docentes, la integración curricular de las TIC los favoreció (gráfico 2) porque el clima escolar cambió en un $22 \%$, generando confianza y entusiasmo, propiciando la comunicación entre docentes y el trabajo en equipo. También se logró el aprendizaje y la apropiación de contenidos por parte de los estudiantes en un $26 \%$, desarrollando competencias en el manejo de las TIC. El cambio más importante fue en las prácticas de enseñanza en un $52 \%$, porque se utilizaron estrategias metodológicas basadas en la fundamentación teórica, el diseño y organización de la información, la aplicación y la socialización, convirtiendo las TIC en una herramienta pedagógica. Se añade que se fomentó la interdisciplinariedad y la transversalidad curricular, se dio la horizontalidad y se propiciaron espacios de reflexión pedagógica.

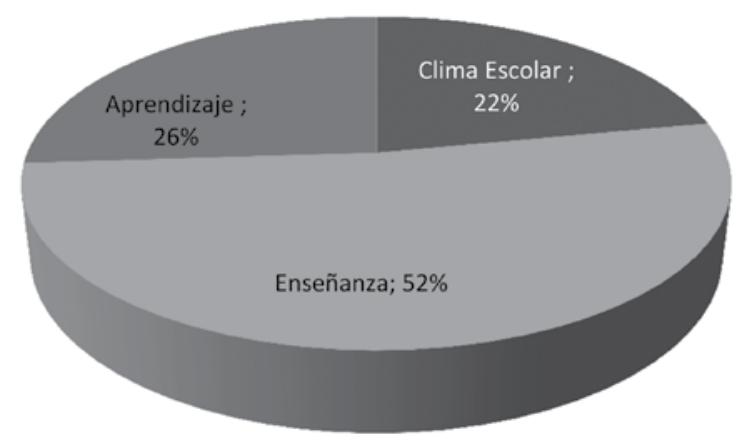

Figura 2. Beneficios para la enseñanza. Datos obtenidos de entrevista a docentes 
Los estudiantes, por su parte, creen que la integración curricular de las TIC les ayudó a mejorar sus aprendizajes en un $41 \%$ porque les permitió desarrollar habilidades de pensamiento, entender, relacionar, profundizar confrontar, intercambiar, atender, aprender y desarrollar competencias al trabajar temas desde la mirada de todas las áreas. Ellos notan que sus conocimientos son más significativos, que aumentó su motivación en un 14\%, que pudieron trabajar con otras áreas del conocimiento experimentando las clases agradables y dinámicas en un $24 \%$. Finalmente las TIC se convirtieron en una herramienta para trabajar a través de las disciplinas (21\%), llevando lo teórico a lo práctico y facilitando su aprendizaje. (gráfico 3).

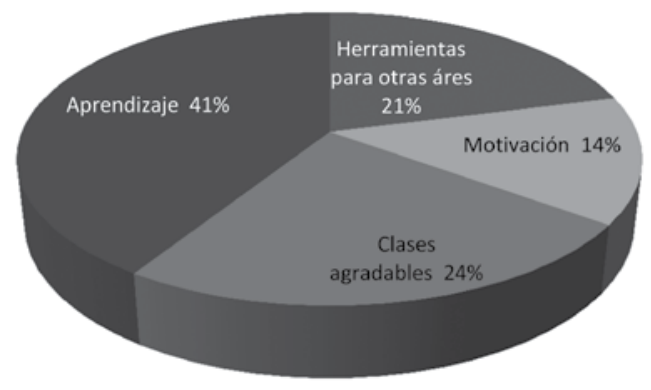

Figura 3. Beneficios para el estudiante. Datos obtenidos según entrevista a estudiantes

Adicionalmente, el $45 \%$ de los estudiantes opinó que las TIC contribuyen a cambiar la metodología para desarrollar mejor las clases por parte de los docentes. El 35\% dijo que hay fomento en la transversalidad y en la relación de contenidos de las áreas, enfocados bajo un tema principal. El 10\% de los docentes se muestran motivados y oxigenados, hecho que hace más dinámicas y agradables las clases. El 10\% de ellos manifestó que ahora se nota el proceso de capacitación ya que hay interés en aprender el uso de las TIC y aplicarlas para enseñar a los estudiantes (gráfico 4).

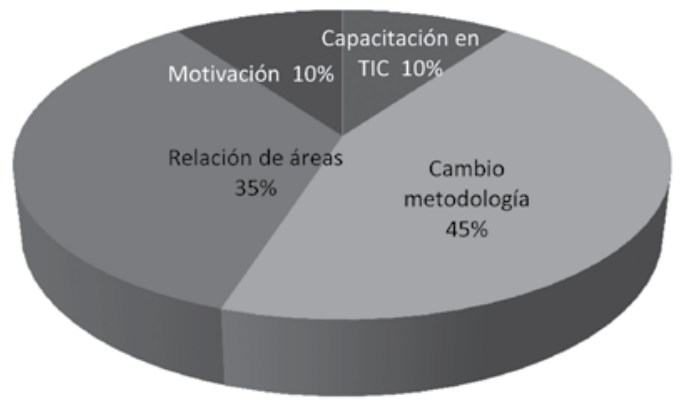

Figura 4. Beneficios para el docente. Datos obtenidos según entrevista a estudiantes 
En relación con el impacto del proyecto en la comunidad educativa (gráfico 5), los entrevistados manifestaron que el proceso de integración curricular de las TIC tuvo un impacto que se discrimina en los siguientes términos: Los docentes en un $46 \%$ mostraron su motivación por el uso de las TIC como herramienta pedagógica que transforma sus prácticas de enseñanza verticales en horizontales, por el incremento del trabajo en equipo y por el dinamismo en las clases. También ven con buenos ojos el intercambio de experiencias, el paso de lo tradicional a lo integrado y el mayor desarrollo de las competencias en el manejo de las TIC.

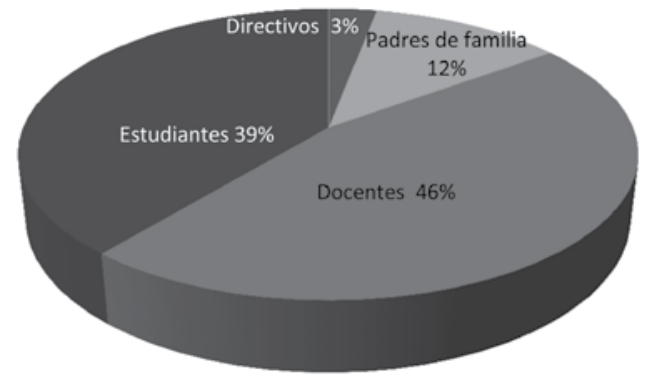

Figura 5. Impacto en la comunidad educativa

El 39\% manifestó que los estudiantes se beneficiaron porque se motivaron aun más por utilizar las TIC en sus procesos de aprendizaje, asistir a clase, trabajar colaborativamente, fomentar la creatividad, socializar sus trabajos, ser protagonistas de su aprendizaje y desarrollar competencias generales y en el uso de las TIC.

El 12\% expresó que el proyecto impactó a los padres de familia por la aceptación del uso de las TIC en las clases, el manejo del lenguaje específico de las TIC y el apoyo a su hijos. La compra de equipos e Internet móvil para uso personal y comunitario, generó una oportunidad de trabajo para muchos habitantes de la región que instalaron negocios relacionados con Internet.

Finalmente, el 3\% manifestó su impacto en los directivos; en menor proporción, por su intervención en la sensibilización a padres de familia, por propiciar espacios de capacitación de docentes y por valorar las TIC como herramienta de aprendizaje, factores estos que de todas maneras resultan muy importantes en el proceso.

Recomendaciones para la integración curricular de las tic en instituciones de educación básica y media

- Estas instituciones deben estar lideradas por docentes en ejercicio porque si estos ayudan a cambiar los escenarios educativos y acceden a ellos, también movilizan estructuras y prácticas institucionales. 
- Se requiere capacitación para utilizar competentemente las nuevas tecnologías.

- El proceso debe hacerse gradualmente como lo propone la FGPU en su modelo de integración y debe partir de los enfoques formulados por los estándares para docentes dados por la Unesco.

- Se sugiere plantear relaciones horizontales, abriendo espacios en donde el docente se capacite, planee, desarrolle y evalúe procesos en torno a una estrategia, que se considera motivadora. En este caso fueron las TIC el pretexto para lograr esas relaciones horizontales en el acto educativo.

- La integración no debe ser impuesta sino concertada, involucrando a todos los docentes y partiendo de las necesidades e intereses de todos.

- Durante el proceso de integración es importante organizar los productos de docentes y estudiantes para evidenciar el desarrollo de las competencias.

- No basta con tener una estrategia de integración de las TIC al currículo; esta deber ser conocida y desarrollada por los docentes y estudiantes que asumen los procesos de enseñanza- aprendizaje en cada una de las áreas y requiere una evaluación y seguimiento continuo.

- Definitivamente, para que la integración de las TIC sea efectiva, se debe partir de las estrategias pedagógicas de los docentes de las demás áreas, no como una simple transferencia sino como elementos potenciadores con las TIC.

\section{Conclusiones}

Para integrar curricularmente las TIC en una institución educativa que ofrezca los niveles de educación básica y media se propone una metodología que parte del diagnóstico de necesidades y niveles de integración curricular de las TIC, la formulación y aplicación de un plan de capacitación, la planeación con unidades integradas, la integración curricular y el seguimiento, evaluación y socialización del proceso, que puede ser modificado por los docentes según las realidades institucionales y contextuales.

El proceso de integración curricular de las TIC se dio en la Institución Educativa Panamericana Puente de Boyacá porque se han cambiado paradigmas en las prácticas pedagógicas y se ha pasado de clases tradicionales a clases activas, dinámicas y constructivas, permitiendo el trabajo en equipo, el aprendizaje dentro y fuera del aula y la horizontalidad pedagógica representada en el cambio en la relación docente-estudiante. 
La nueva cultura institucional ha puesto en escena espacios donde otros docentes y estudiantes de los demás grados sugieren continuar con este proceso e incluirlo en otras áreas pese a las dificultades de recursos y a las condiciones culturales, evidenciando de esta forma, una innovación.

Los docentes concluyen que el proceso de integración de las TIC al currículo es una "propuesta que contribuye al acercamiento de los docentes y los nuevos estudiantes del siglo XXI para quienes la tecnología es su "modus vivendi", promueve el mejoramiento en el quehacer docente puesto que implementa en su área una herramienta educativa de gran interés para el estudiante, motivándolo para el desarrollo de las diferentes actividades y facilita el aprendizaje en el estudiante logrando mayor comprensión de los temas, haciéndolos significativos". La integración curricular de las TIC en educación media se debe a la claridad en la conceptualización de las TIC, currículo, currículo integrado, integración curricular de las TIC, estrategias de aplicación del proceso, formulación de la propuesta metodológica, impacto generado e incluso, algunas sugerencias a tener en cuenta para que la integración sea más efectiva.

\section{Referencias bibliográficas}

Cabello, R. (coord) (2006). "Yo con la computadora no tengo nada que ver". Buenos Aires, Prometeo.

Cabello \& Aprea. (2004).Tecnologías de la comunicación, Educación y desarrollo, en Carniglia, E. y Cimadevilla, G. (edit), Comunicación, ruralidad y desarrollo: Mitos, paradigmas y dispositivos del cambio, Buenos Aires, Ediciones INTA.

Camargo. (2007). Las Tic en el currículo. Consultado el 12 de marzo de 2009 en http:// ticenelaula.espacioblog.com/ post/2007 / aaque-son-TIC- Bogotá, Colombia.

Dede. (2000). Aprendiendo con tecnología. Barcelona, Paidós.

Díaz. (2006). La educación en valores: Avatares del currículum formal, oculto y los temas transversales. Revista Electrónica de Investigación Educativa, 8 (1). Consultado el 16 de marzo de 2010 en: http://redie.uabc.mx/vol8no1/contenido-diazbarriga2.html.

Dockstader. (1999). Teachers of the XXI century know the what, why, and how of technology integration. T.H.E. Journal, pp. 73-74, January.

Echeverría. (2000). Educación y Tecnologías Telemáticas. Revista Iberoamericana. núm. 24. Organización de estados iberoamericanos para la educación, la ciencia y la cultura. Consultado el 16 de marzo de 2010 en http://www.rieoei.org/rie24a01.htm 
Escudero. (1981). Modelos Didácticos. Barcelona, Oikos-Tau, 1981.

Fogarty. (1991). Ten ways to integrate the curriculum. Educational Leadership, October, pp. 61-65.

Fundación Gabriel Piedrahíta Uribe. (2008). Modelo para integrar TIC en el currículo. Consultado el 12 de febrero de 2008 en www.eduteka.org/tema_mes. php3?TemaID=0017.

Galvis. (2001). Aprender y enseñar en compañía y con apoyo de TIC, tecnologías de información y de comunicaciones. Consultado el 30 de mayo de 2009 en http://www. metacursos.com/documents/Aprender_enseniar_en_compania.pd.

Gimeno. (1981). Teoría de la enseñanza y desarrollo del currículum. Madrid, Anaya.

González. (2000). Modelos pedagógicos para un ambiente de aprendizaje en NTIC.

Conexiones, Informática y Escuela. Un Enfoque global. Consultado en diciembre 5 de 2008 en http://www.colombiaaprende.edu.co/html/sitios/1610/articles-131558 pdf1.pdf.

Grabe, \& Grabe. (1996). Integrating technology for meaningful learning. Boston, Houghton Mifflin.

Gross. (2000). El ordenador invisible: hacia la apropiación del ordenador en la enseñanza. Barcelona, Editorial Gedisa.

Gutiérrez. (2007). Integración curricular de las TIC y educación para los medios en la sociedad del conocimiento. OEI - Revista iberoamericana de educación, núm 45, 2007. Consultado el 16 de marzo de 2009 en http://www.rieoei.org/rie45a06.htm.

Herrera. (2008). Propuesta de un modelo de integración curricular de las TIC. Cibersociedad. Universidad Tecnológica Metropolitana. Consultado el 6 de noviembre de 2009 en www.cibersociedad.net/public/documents/71_vd24.doc - España.

Hopenhayn. (2003). Educación, comunicación y cultura en la sociedad de la información: una perspectiva latinoamericana. Revista de la CEPAL. núm. 81. Consultado el 14 de junio de 2008 en www.eclac.org/publicaciones/xml/8/11678/ Icl1844e.pdf.

Hurtado. (2002). Integración curricular de las tecnologías de la comunicación y la información. C.P.E.E. Pérez Urruti. Churra-Murcia. Consultado el 18 de agosto de 2010 en http://www.tecnoneet.org/docs/2002/5-52002.pdf. 
Jacobs, H. H. Ed. (1991). Curriculum integration, critical thinking and common sense. Cogitare.

Johnson. (1967). Definitions and models in curriculum theory. International Review of Education, 19, pp. 187-194.

Jonassen \& Reeves. (1996). Learning with technology: Using Computers as cognitive tools. In D.H. Jonassen (Ed), Handbook of Research for Educational Communications and Technology (pp. 693-719). New York, Macmillan. Consultado el 15 de marzo de 2010 en http://www.eduteka.org/imprimible.php?num=78.

Magendzo. (2003). Transversalidad y currículo. Cooperativa Editorial Magisterio, Bogotá. Marqués. (2000). Las TIC y sus aportaciones a la sociedad. Consultado el 2 de abril de 2009 en http://www.pangea.org/peremarques/tic.htm® 2000.

Merrill, Hammons, Vincent, Reynolds, Cristiansen \& Tolman. (1996) (Third Edition). Computers in Education. Boston, Allyn \& Bacon.

Montenegro. (2005). Gestión Pedagógica: Currículo de Educación Formal. Supervisor de Educación del Distrito Capital. Doctor en Educación. imonten@ hotmail.com. Bogotá.

Ortiz. (2006). Retos y perspectivas del currículo integrado. Cuaderno de Investigación en la Educación, 21, pp. 35-56. Consultado el 13 mayo de 2009 en cie.uprrp.edu/ cuaderno/.../21/02a.html - Puerto Rico.

Pedraza \& Lara. (2007). Integración curricular de las TIC en la educación superior. Guía de orientación para la integración de las TIC en educación superior. Grupo de Estudio No. 4 de la Red Virtual de Tutores. Consultado el 12 de Febrero de 2009 en http://www. tutorvirtual.utp.edu.co/.../file.../Integracion_Curricular_GE4.pdf.

Porlán. (1993). Constructivismo y escuela. Hacia un modelo de enseñanza-aprendizaje basado en la investigación. Sevilla, Díada.

Rosario. (2005). La tecnología de la información y la comunicación (TIC). Su uso como herramienta para el fortalecimiento y el desarrollo de la educación virtual. CiberSociedad Consultado el 24 de mayo de 2009 en http://www.cibersociedad.net/ archivo/articulo. php?art=218.

Sánchez, J. (2002). Integración curricular de TIC. Concepto e ideas. [En línea]. Revista Enfoques educacionales. $6^{\circ}$ Congreso Iberoamericano, $4^{\circ}$ Simposio Internacional de Informática Educativa, $7^{\circ}$ Taller Internacional de Software Educativo: IE-2002: Vigo, 20, 21, 22 de noviembre de 2002. Consultado el 17 de septiembre de 2009. Disponible en: http://lsm.dei.uc.pt/ribie/docfiles/txt2003729191130paper-325.pdf. 


\section{Dora Lidia Orjuela Forero}

Integrar las TIC al currículo en la educación media, artículo producto de la investigación

Stenhouse. (1991). Investigación y desarrollo del currículum. Tercera Edición. Madrid: Morata.

Unesco. (2008). Estándares de Competencias en TIC para docentes. consultado el 15 de agosto de 2009 de http://portal.unesco.org/es/ev.php-url_id=41553\&url do=do_ topic\&url_section=201.html, http://cst.unesco-ci.org/sites/projects/cst/ default. aspx. Londres. 2008. 